\title{
REVISIONES
}

\section{Motivación escolar: Claves para la formación motivacional de futuros docentes*}

\author{
School motivation: Keys to future teachers' motivational training \\ Motivação escolar: Chaves para a formação motivacional de futuros professores
}

\author{
Jorge Valenzuela, ${ }^{a}$ Carla Muñoz Valenzuela ${ }^{b}$ Ilich Silva-Peña, ${ }^{c}$ \\ Viviana Gómez Nocetti, ${ }^{\text {Andrea Precht Gandarillas. }}{ }^{\text {. }}$ \\ a Centro de Estudios Avanzados, Universidad de Playa Ancha, Chile. Fono: 032-2624710. \\ Correo electrónico: jorge.valenzuela@upla.cl

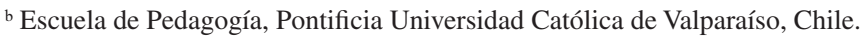 \\ Correo electrónico: carla.munoz@gmail.com \\ c Facultad de Educación, Universidad Católica Silva Henríquez, Chile. \\ Correo electrónico: ilichsp@gmail.com \\ d Campus Villarrica, Pontificia Universidad Católica, Chile. \\ Correo electrónico: vgmezn@uc.cl \\ e Escuela de Educación, Universidad Santo Tomás, Sede Talca, Chile. \\ Correo electrónico: aprecht@santotomas.cl
}

\begin{abstract}
RESUMEN
El presente artículo sugiere esquemas teóricos que parecieran ser más pertinentes para la formación motivacional de futuros profesores. Ello surge de la constatación de que la formación motivacional de los estudiantes de pedagogía muchas veces se hace a partir de modelos teóricos que si bien pueden ser muy predictivos, no son lo suficientemente adecuados para proponer pistas que focalicen la intervención pedagógica en la motivación por el aprendizaje. Se discuten distintas aproximaciones teóricas reconociendo sus aportes y limitaciones y se propone, finalmente, un esquema teórico que puede contribuir de manera pertinente a que los futuros docentes tengan elementos teóricos sobre los cuales sostener sus esfuerzos por motivar a sus alumnos, focalizándose en los factores motivacionales del aprendizaje y dejando atrás nociones generales o teóricamente poco pertinentes para este fin.
\end{abstract}

Palabras clave: motivación, motivación escolar, formación docente, profesorado, teoría motivacional.

\begin{abstract}
This article suggests theoretical schemes that seem most relevant to motivational training of future teachers. This arises from the observation that pedagogy students' motivational training is often made from theoretical models that, while they may be very predictive, are not adequate enough to offer clues for pedagogical intervention that focus on motivation for learning. Different theoretical approaches are discussed recognizing their contributions and limitations. Finally, we propose a theoretical framework that can contribute, in a relevant way for future teachers, to acquire theoretical elements which sustain their efforts to motivate their students, focusing on learning the motivational factors and leaving behind notions theoretically less relevant for this purpose.
\end{abstract}

Key words: motivation, school motivation, teacher training, teacher, theory.

Este artículo forma parte del proyecto FONDECYT Regular $2012 \mathrm{~N}^{\circ}$ 1120351: "Estudio de las Representaciones de la Motivación Escolar, Autoeficacia y Sentido del Aprendizaje Escolar: Aportes para el Mejoramiento de la Formación Motivacional de los Docentes". 


\section{RESUMO}

Este artigo sugere esquemas teóricos que parecem ser mais relevantes para a formação motivacional de futuros professores. Surge da constatação de que a formação dos estudantes da Pedagogia, muitas vezes, se dá a partir de modelos teóricos que, embora possam ser muito previsíveis, não são suficientemente adequados para propor pistas que focalizem a intervenção pedagógica na motivação da aprendizagem. Reconhecendo suas contribuições e limitações, diferentes abordagens teóricas são discutidas e, finalmente, propõe-se um esquema teórico que pode contribuir, de maneira importante, para que futuros professores tenham elementos teóricos sobre os quais sustentem seus esforços para motivar seus alunos, focalizando nos fatores motivacionais da aprendizagem e desprezando noções gerais e teoricamente pouco pertinentes para esta finalidade.

Palavras chave: motivação, motivação escolar, formação de professores, professores, teoria motivacional.

\section{MOTIVACIÓN Y CONTEXTO ESCOLAR}

La literatura ha mostrado consistentemente un efecto significativo de la motivación en los procesos de aprendizaje (Delobbe, 2007; Long, Monoi, Harper, Knoblauch \& Murphy, 2007; Robbins et al., 2004; Huertas, 1997; Utman, 1997). La diversidad de formas de entender la motivación responde tanto a perspectivas teóricas, como a preguntas e intencionalidades diferentes (Miñano Pérez y Castejón Costa, 2008; Boekaerts, Smelser \& Baltes, 2001; Gollwitzer, Oettingen, Smelser \& Baltes, 2001; Murphy y Alexander, 2000). Algunos intentan dar cuenta de la naturaleza del fenómeno en general (e.g. Nuttin, 1985), otros son más específicos y aplicables al contexto general que nos interesa: el aprendizaje académico, y más específicamente, el aprendizaje académico a nivel escolar. Ejemplo de ello es la teoría de la autodeterminación (Vansteenkiste, Lens \& Deci, 2006; Ryan \& Deci, 2000; Pelletier et Vallerand, 1993; Deci, Vallerand, Pelletier \& Ryan, 1991).

Normalmente cuando se habla de motivación escolar se hace referencia a aquella motivación que impulsa al estudiante a realizar una serie de tareas que los profesores le proponen como mediación para el aprendizaje de los contenidos curriculares. Sin embargo, la motivación escolar si bien comprende la realización de la tarea, no se agota en ella. En términos más precisos, la motivación escolar tiene que ver más concretamente con la activación de recursos cognitivos para aprender aquello que la escuela propone como aprendizaje (Valenzuela, 2007), y no sólo con querer realizar una tarea específica en un momento dado. En esta dirección, la pregunta por la motivación tiene que ver, en términos generales, con el valor de la tarea y con el sentimiento de competencia frente a ella, pero también, a nivel de la actividad general, con los motivos que dan sentido a la activación de dichos recursos cognitivos en pos del aprendizaje (motivos para aprender).

Existen variadas perspectivas para abordar la motivación escolar. Hay algunas opciones teóricas que se destacan por su capacidad predictiva. Por ejemplo, la conceptualización de la motivación que distingue motivación intrínseca y extrínseca (Sansone \& Harackiewiecz, 2000; Deci \& Ryan, 1999, 1985; Deci et al., 1991) ha mostrado ser un enfoque teórico que permite predecir de manera significativa el desempeño académico. Sin embargo, este tipo de enfoques tiene la debilidad de que, así conceptualizada la motivación, el margen de acción que queda para una intervención a nivel escolar es muy limitado. Si lo que mejor predice el desempeño es tener una motivación intrínseca, ¿cómo puede un profesor actuar sobre ella intencionadamente? Si ella es intrínseca, a lo más podemos generar condiciones que favorezcan su surgimiento. Lo anterior es también válido, a nuestro juicio, para otras opciones teóricas que, a pesar de sus virtudes, no aportan pistas más claras en vistas a la intervención. 
Algo similar ocurre con la teoría de metas, ampliamente difundida en el medio escolar (Elliot \& Dweck, 2007; Alonso Tapia, 2005, 1997; Dweck \& Leggett, 1988), sobre todo en lo referido al tipo de metas. La investigación ha sido consistente en mostrar que los alumnos que poseen metas de aprendizaje aprenden más que aquellos cuyas metas se orientan al desempeño (aprobar el curso, por ejemplo). Sin embargo, no es claro cómo se logra que los alumnos "motivados" por el desempeño lleguen a una motivación orientada al aprendizaje. En este sentido, ciertamente no basta con que los profesores les digan a sus alumnos que el esfuerzo escolar es para aprender y no sólo para aprobar la materia.

Otro tanto ocurre con la teoría atribucional de motivación de logro de Bernard Weiner (Weiner, 2008, 1990, 1985; Alonso Tapia, 2007; García López, 2006; Barca Lozano, Peralbo Uzquiano y Breñilla Blanco, 2004; Crespo, 1982), según la cual aquellos estudiantes con determinados tipos de atribuciones sobre su éxito escolar, específicamente atribuciones internas, controlables y modificables (característicamente la atribución al esfuerzo), obtienen mejores resultados instruccionales (e.g. Miranda, Arlandis y Soriano, 1997). En este caso, aunque existen programas de entrenamiento (re)atribucional que pueden ser un aporte interesante, esto no soluciona el problema de que el estudiante quiera, efectivamente, aprender.

\section{EL APRENDIZAJE ESCOLAR}

La dificultad de aplicar la teoría motivacional al aula es que el contexto escolar tiene unas ciertas características, las que muchas veces por sabidas son olvidadas, que no obstante forman parte de la especificidad del aprender en la escuela. El aprendizaje escolar tiene como foco la apropiación de un conocimiento que depende de lo disciplinar (matemáticas, ciencias, etc.), sin necesariamente ser igual a éste (Cfr. Astolfi, 2003; García, 1997; Delbos et Jorion, 1984). Si bien el proceso de aprendizaje escolar es una construcción de conocimiento, debe, de alguna manera, coincidir con el conocimiento disciplinar que se intenciona.

Por otra parte, y a diferencia de otros contextos en donde lo que va a ser aprendido es, en alguna medida, elegido por el estudiante, en la escuela el alumno tiene que aprender obligatoriamente una serie de contenidos. No tiene otra opción que aprender (de manera más o menos profunda, ésa es otra cuestión), si desea aprobar. Reconocemos que puede haber formas de hacer sentir al alumno que él ha elegido, de algún modo, dichos contenidos, aportando un sentimiento de autodeterminación frente a la tarea y, con esto, mejorar el compromiso con la tarea... pero al fin de cuentas, son otros los que deciden qué es lo que el estudiante debe aprender.

Adicionalmente, constatamos, como veremos más adelante, que a los profesores se les exige "motivar a sus alumnos", dado que ellos tienen una responsabilidad específica en la facilitación de dichos aprendizajes. Lo cierto es que no siempre han recibido las herramientas adecuadas para hacerlo (Silva-Peña, Navarrete y Zúñiga, 2012).

Por todo lo anterior, la especificidad del contexto escolar exige que la perspectiva teórica aporte no sólo elementos predictivos en relación al desempeño (aprendizaje), sino que, además, la perspectiva teórica proporcione pistas para que los docentes puedan integrar la dimensión motivacional dentro del diseño instruccional, estimulando así la motivación por aprender los contenidos curriculares de la escuela. 


\section{MOTIVACIÓN ESCOLAR}

En este contexto, nuestra opción teórica incorpora dos esquemas teóricos complementarios. El primero tiene que ver con la tarea propiamente tal, inspirada en el modelo de expectación/valor propuesto por Jacqueline Eccles y Allan Wigfield (Neuville, 2004; Neuville, Bourgeois \& Frenay, 2004; Wigfield \& Eccles, 2000, 1992; Wigfield, 1994; Eccles et al., 1983). Este modelo sostiene que la motivación por el logro de una tarea sería el producto de la expectativa más el valor asignado a la tarea.

Por un lado, el primer elemento corresponde a la expectativa que una persona tiene de poder realizar adecuadamente una tarea. Esta noción de expectativas se distingue conceptualmente de las creencias de autoeficacia (Bandura, 1997, 1986, 1977; Pajares, 1996). La expectativa se centra en competencias futuras mientras que el constructo -ya clásico de Bandura- se focaliza en el presente. Sin embargo, y dada la íntima correlación entre ellas (Wigfield, Eccles \& Roeser, 1998; Bandura, Barbaranelli, Caprara \& Pastorelli, 1996; Pajares, 1996; Eccles et al., 1983), en la práctica serían equivalentes. Por esta razón, este modelo integra también los aportes que Bandura ha realizado a la motivación.

Por otro lado, junto a la expectativa, encontramos el valor que es asignado a la tarea. El valor de la tarea estaría compuesto por cuatro componentes: la importancia, el interés, la utilidad y el costo (Cfr. Neuville, Frenay \& Bourgeois, 2007; Wigfield \& Eccles, 1992; Eccles et al., 1983). La importancia (attainment value) corresponde a cuán relevante es para el sujeto realizar bien una determinada tarea. El interés (intrinsic value) corresponde al disfrute/gusto (enjoyment) por hacer la tarea. Este componente retoma los aportes de Deci \& Ryan (1999, 1985; Deci et al., 1991; Vansteenkiste et al., 2006) que ponen de relieve que cuando la tarea es valorada intrínsecamente, existen importantes consecuencias psicológicas que repercuten positivamente en el desempeño. Expresado de otro modo, este componente de la motivación corresponde al interés que se suscita en el sujeto (Schiefele, 1991; Cfr. Wigfield \& Cambria, 2010; Silvia, 2006). Por su parte, la utilidad percibida de la tarea (utility value) se refiere a en qué medida una tarea se adecua a los planes futuros de la persona. Finalmente, el costo (cost) alude a la percepción del sujeto respecto de cuánto deberá sacrificar o invertir para realizar la actividad (Anderson, 2000). Pese a que los trabajos de Jacqueline Eccles y su equipo se han concentrado en los tres primeros componentes, Neuville, Bourgeois \& Frenay (2004) proporcionan evidencia empírica a través de análisis factoriales confirmatorios para demostrar su pertenencia al constructo "valor", corroborando así el modelo (Neuville, 2004).

Una segunda perspectiva, que complementa la anterior, focaliza la motivación (ya no en el plano de la tarea, sino en la actividad general) en los motivos que dan sentido a aprender en la escuela, dicho de otra manera, en los motivos que contextualizan y favorecen la activación de los recursos cognitivos para aprender lo que la escuela quiere enseñar. La experiencia pedagógica y la evidencia científica muestran que un estudiante puede querer realizar la tarea escolar por muy diversos motivos o razones. Estas razones van desde la evitación (e.g. motivación puntual como realizar la tarea para salir a recreo, o a más largo plazo, para que los padres o el profesor lo dejen en paz), hasta motivos de verdadero interés por el aprendizaje.

Tradicionalmente, esta cuestión ha sido abordada principalmente desde la teoría de metas (Matos y Lens, 2006; Elliott, Shell, Henry \& Maier, 2005; Alonso Tapia, 1997; González Cabanach, Valle, Núñez y González-Pienda, 1996; Ames, 1992), distinguiendo 
diferentes orientaciones en la realización de la tarea (metas de aprendizaje/metas de desempeño). Sin embargo, esta perspectiva, aun cuando tiene bondades predictivas, se mantiene en el plano de la tarea (bajo qué lógica el estudiante aborda el trabajo escolar), y no focaliza el análisis al interior de las metas de aprendizaje. Quizá una aproximación interesante es el trabajo de Anne Marie Conley (2012), quien trata de integrar la teoría de metas con los modelos de expectativa/valor, aportando una mirada más compleja a la motivación de logro. No obstante, desde la educación requerimos de modelos que, como decíamos antes, no sólo sean predictivos a nivel de la tarea, sino que den pistas a los educadores para facilitar la motivación escolar, entendida como querer aprender.

Así, en el contexto escolar, la pregunta motivacional no está sólo referida a por qué los alumnos querrían realizar las tareas propuestas por el profesor, sino que además, debemos complementarla con la pregunta sobre por qué los estudiantes querrían aprender dichos contenidos (por qué activarían sus recursos cognitivos para aprender). Desde esta perspectiva, Valenzuela $(2009,2007,2006)$ ha propuesto una clasificación de tipos de motivos que serían el marco a partir del cual los estudiantes otorgarían sentido a aprender en la escuela. Estos motivos se agrupan en torno a: a) la responsabilidad social, b) el desarrollo personal, c) la movilidad social, d) la sobrevivencia y e) el "mal menor". Estos tipos de motivos son valorados diferencialmente por los estudiantes, y su potencial efecto dependería de la validación que sus pares y, especialmente, sus profesores hacen de dichos motivos.

En síntesis, entender la motivación escolar pasa por comprender tanto la motivación a la tarea como la motivación a la actividad. Y en la complementariedad de ambas dimensiones, debemos buscar las pistas para una intervención más efectiva.

\section{PROFESORES Y MOTIVACIÓN}

Es claro que una de las exigencias que se hace al profesor es la de "motivar a sus alumnos" para el aprendizaje. Esta exigencia se hace desde una posición fuera del aula, asumiendo que se trata de una tarea básica en el oficio docente que debería formar parte de los saberes profesionales de cualquier profesor, obviando la complejidad del asunto y lo que implica la preparación para ello.

Tan complejo es motivar a los alumnos que, en el caso de Chile, los documentos oficiales (MINEDUC, 2003), cuando se aborda el tema de la motivación como competencias de los docentes (acordadas por los gremios de profesores y el Ministerio de Educación), se limitan a señalar que los docentes deben tener "actitudes explícitas de interés por el aprendizaje, la indagación y la búsqueda y de autosatisfacción cuando llega[n] a resultados adecuados", y generar "estilos de trabajo en que las preguntas, aportes y experiencias de todos los alumnos son incorporados, y no sólo las de aventajados o con mejores rendimientos" (MINEDUC, 2003: 23). De este modo, se apunta a reforzar ciertas actitudes del profesor y la construcción de un clima de clase que dé seguridad a los alumnos y, por tanto, un ambiente propicio para la participación. Claramente esto es una condición necesaria, pero no suficiente para estimular a los estudiantes a querer aprender.

No existe en la actualidad información específica sobre cómo está siendo abordada la formación motivacional de los futuros docentes. Con todo, hay pistas (Silva-Peña et al., 2012) que sugieren que la noción de motivación que subyace a la formación de nuestros 
futuros docentes está fuertemente influida por las perspectivas clásicas de la motivación, en concreto, por la distinción intrínseco/extrínseco (Vansteenkiste et al., 2006; Sansone \& Harackiewiecz, 2000; Deci \& Ryan, 2000, 1999, 1985; Cameron \& Pierce, 1994), por la creencia de que en la medida que los docentes tengan altas expectativas de sus alumnos ellos aprenderán más (Weinstein, Marshall, Sharp \& Botkin, 1987; Feldman \& Theiss, 1982; Feldman \& Prohaska, 1979), y por la teoría de aprendizaje significativo de Ausubel (1978), quien sostiene que se debe vincular el nuevo contenido con experiencias previas de los alumnos.

El problema de la primera influencia estriba básicamente en las posibilidades de maniobra para intervenir pedagógicamente, si lo deseable es la motivación intrínseca ¿cómo "motivar" sin que ello se transforme en una influencia "extrínseca"? En relación con las expectativas del profesor y el efecto Pigmalión, si bien constatamos la relación entre las variables, no es del todo claro cómo un profesor puede tener o hacer persistir las altas expectativas (verdaderas) cuando la información que recibe del propio alumno o del contexto socioeconómico/cultural van en sentido contrario.

Frente al tercer caso, la estrategia de conectar los nuevos contenidos con experiencias previas del alumno parece razonable y necesaria (para el caso del aprendizaje por recepción), el problema es que no siempre se establece la conexión deseada, ya que el objeto puede ser leído bajo una lógica diferente por el profesor y por el alumno. Ejemplo de esto último es cuando se toma la cordillera como objeto de estudio (orogénesis, estructura, etc.): para el profesor se trata de un objeto de estudio mientras que para el alumno sólo es un lugar de paseo. Entonces, ¿por qué haber disfrutado de la cordillera como lugar de paseo motivará a un alumno a querer aprender sus aspectos orogénicos e identificar correctamente sinclinales y anticlinales?

Los tres aspectos mencionados son, ciertamente, importantes y necesarios de considerar, ya que pueden contribuir a un mejor aprendizaje, pero se corre el serio riesgo de concebirlos como suficientes para la motivación de los alumnos. Parece claro que una formación motivacional adecuada debería entregar una serie de conocimientos disciplinares que ayuden a comprender el fenómeno de la motivación escolar y que den pistas para el diseño de estrategias integradas en el diseño de la clase. Este tipo de formación, además, debiera verse refrendada en un sentimiento de competencia de los docentes frente al desafío de ayudar a motivar a sus alumnos (autoeficacia motivacional).

\section{ALGUNAS CLAVES PARA LA FORMACIÓN MOTIVACIONAL DE FUTUROS DOCENTES}

Desde el sentido común podríamos señalar que los profesores debieran ser capaces de facilitar la motivación de los alumnos hacia el aprendizaje. Sin embargo, no basta con exigirlo, hay que formar motivacionalmente a los futuros docentes. No basta con el carisma personal, es necesario contar con conocimientos y con una conceptualización adecuada de la motivación que sirva como marco y guía de los esfuerzos motivacionales de los docentes. Por lo anterior, y dada la multiplicidad de enfoques sobre la motivación, se hace necesario avanzar en un análisis más fino que nos permita aproximarnos al fenómeno de la motivación con un andamiaje teórico adecuado para visualizar estrategias en la formación inicial, las cuales proporcionen herramientas para la intervención en pos de un mejor aprendizaje. 
Desde esta perspectiva, hemos querido relevar algunas aproximaciones teóricas que pueden arrojar luz sobre cómo, desde la docencia, entender la motivación por el aprendizaje de los alumnos. A partir de allí es posible repensar diseños que contribuyan, apoyen o fomenten la motivación por el aprendizaje.

La formación motivacional de los futuros docentes debiera ser sólida y pertinente. La forma en cómo las personas se representan la realidad (representaciones sociales, teorías implícitas, etc.), condiciona y contextualiza, de alguna manera, sus prácticas sociales (Abric, 2001a, 2001b; Flament, 2001). En este contexto, la representación y los conocimientos teóricos que tenga el profesor sobre la motivación en el contexto escolar son relevantes para su práctica pedagógica. Lo son en la medida en que su concepción le permite visualizar estrategias y acciones concretas dentro de su diseño instruccional. Si para él/ella, por ejemplo, la motivación es intrínseca/extrínseca, es poco el margen de maniobra que le queda para facilitar motivacionalmente el aprendizaje. Evidentemente, una concepción determinada de la motivación no asegura la puesta en ejecución de prácticas motivacionales consistentes, pero es el marco donde ellas son posibles.

En esta línea, si queremos que nuestros profesores medien motivacionalmente el aprendizaje escolar de manera efectiva, deben tener nociones adecuadas de cómo funciona la motivación de los alumnos en el contexto escolar, es decir, la motivación por aprender, no sólo la motivación por realizar la tarea. En esta dirección, lo importante es que la teoría les provea pistas para el diseño y la puesta en ejecución de estrategias, así como para la toma de decisiones pedagógicas que contribuyan a la motivación por el aprendizaje de sus alumnos, de manera tal que sea posible ir más allá de una conceptualización de la motivación construida desde el sentido común.

Aunque esta propuesta está lejos de ser definitiva, pretende ser un incentivo para tomar en serio la formación motivacional que estamos dando a nuestros futuros maestros. En aras de aportar a la formación motivacional de los futuros profesores, presentamos a manera de síntesis los siguientes puntos que pueden ser considerados:

1. No basta con exigir al profesor que motive a los alumnos, hay que darle elementos teóricos para que planifique, gestione y tome decisiones pedagógicas que contribuyan a este fin.

2. No debemos olvidar que el foco de la motivación escolar debe estar puesto en el aprendizaje. Aunque parezca obvio, hay una tendencia a superponer los focos de amenizar la clase y el de lograr que se realice la tarea, por sobre esperar que el alumno quiera aprender. Claramente estos focos son concurrentes y deseables, pero no pueden hacernos perder de vista que lo central es que el estudiante active sus recursos cognitivos para aprender de manera profunda.

3. Dentro de la formación pedagógica debemos tener la capacidad de no sólo dar cuenta de teorías de la motivación en general, sino que debemos ser capaces de aquilatar su aporte desde los desafíos pedagógicos, esto es, mostrar cómo una teoría, al tratar de explicar la realidad, le da pistas al docente para que a través de su intervención contribuya a que el estudiante quiera aprender.

4. Desde esta perspectiva, creemos importante que la formación docente vaya más allá de los esquemas clásicos de la teoría de la autodeterminación (motivación intrínseca/extrínseca), y se integren, a nivel de la tarea, esquemas teóricos como los propuestos por Eccles \& Wigfield (2002; Wigfield \& Eccles, 2000), que se ajusten las exigencias de la tarea (García Bacete y Doménech Betoret, 1997), teniendo cuidado de los impactos que puede generar una atribución inadecuada al éxito y especialmente al fracaso de una tarea. 
5. Finalmente, es importante integrar dentro de la formación motivacional esta dimensión más general que va más allá de la tarea y a la que llamamos actividad. En esta línea, tenemos que tomar conciencia de que no basta con que el estudiante quiera hacer la tarea; es crucial que la actividad global tenga sentido para el estudiante, y que el profesor pueda conectarse con aquellos motivos del alumno que pueden dar significado a la activación de los recursos cognitivos para aprender. Evidentemente, lo anterior no implica abdicar frente a la lógica del estudiante y sus motivos para aprender, sino tomar esos motivos como un punto inicial a partir del cual se puede ampliar el horizonte de sentido de los aprendizajes propuestos. Esto pasa, ciertamente, por cuestionar la creencia ingenua de los futuros profesores en torno a que los únicos motivos válidos para aprender son los del docente, los cuales serían, naturalmente, compartidos por los estudiantes.

\section{REFERENCIAS BIBLIOGRÁFICAS}

Abric, J. C. (2001a). Les représentations sociales: Aspects théoriques. En J. C. Abric (Ed.), Pratiques sociales et représentations (pp. 11-36). Paris: Presses Universitaires de France.

(2001b). Pratiques sociales, représentations sociales. En J. C. Abric (Ed.), Pratiques sociales et représentations (pp. 217-238). Paris: Presses Universitaires de France.

Alonso Tapia, J. (2007). Atribución de la causalidad y motivación del logro: Estudio evolutivo de la utilización de información en la utilización de juicios de atribución. Estudios de Psicología, (16), 13-27.

(2005). Motivaciones, expectativas y valores-intereses relacionados con el aprendizaje: El cuestionario MEVA. Psicothema, vol.17 (3), 404-411. (1997). Motivar para el aprendizaje. Teoría y estrategias Barcelona: Edebé.

Ames, C. (1992). Classrooms: Goals, Structures, and Student Motivation. Journal of Educational Psychology, vol.84 (3), 261-271.

Anderson, P. (2000). Cost Perception and the Expectancy-value Model of Achievement Motivation. Annual Meeting of the American Educational Reaserch Asociation. New Orleans, United States.

Astolfi, J. P. (2003). Aprender en la escuela. Santiago: LOM.

Ausubel, D. P. (1978). Educational Psychology: A Cognitive View. New York: Rinehart \& Winston.

Bandura, A. (1997). Self-efficacy: The Exercise of Control. New York: W. H. Freeman and Company.

, Barbaranelli, C., Caprara, G. \& Pastorelli, C. (1996). Multifaceted Impact of Selfefficacy Beliefs on Academic Functioning. Child Development, vol.67 (3), 1206-1222. (1986). Social Foundations of Thought and Action. New York: Prentice-Hall. (1977). Self-efficacy: Toward a Unifying Theory of Behavioral Change. Psychological Review, vol.84 (2), 191-215.

Barca Lozano, A., Peralbo Uzquiano, M. y Breñilla Blanco, J. (2004). Atribuciones causales y enfoques de aprendizaje. Psicothema, vol.16 (1), 94-103.

Boekaerts, M., Smelser, N. J. \& Baltes, P. B. (2001). Motivation, Learning, and Instruction. In N. J. Smelser \& P. B. Baltes (Eds.), International Encyclopedia of the Social and Behavioral Sciences (pp. 10112-10117). Oxford: Elsevier.

Cameron, J. \& Pierce, W. D. (1994). Reinforcement, Reward, and Intrinsic Motivation: A Metaanalysis. Review of Educational Research, vol.64 (3), 363-423.

Conley, A. M. (2012). Patterns of Motivation Beliefs: Combining Achievement Goal and Expectancy-value Perspectives. Journal of Educational Psychology, vol.104 (1), 32-47. 
Crespo, E. (1982). Los procesos de atribución causal. Estudios de Psicología, (12), 33-45.

Deci, E. L. \& Ryan, R. M. (2000). The "What" and "Why" of Goal Pursuits: Human Needs and the Self-determination of Behavior. Psychological Inquiry: An International Journal for the Advancement of Psychological Theory, vol.11 (4), 227-268.

(1999). A Meta-analytic Review of Experiments Examining the Effects of Extrinsic

Rewards on Intrinsic Motivation. Psychological Bulletin, vol.125 (6), 627-668.

Plenum.

(1985). Intrinsic Motivation and Self-determination in Human Behavior. New York:

Vallerand, R. J., Pelletier, L. G. \& Ryan, R. M. (1991). Motivation and Education: The

Self-determination Perspective. Educational Psychologist, vol.26 (3/4), 325-346.

Delbos, G. et Jorion, P. (1984). La transmission des savoirs. Vol. 2. Paris: Editions de la Maison des Sciences de l'Homme.

Delobbe, N. (2007). Facteurs de motivation et de transfert d'apprentissage en formation: Une étude séquentielle dans le contexte d'une formation au leadership. Psychologie du Travail et des Organisations, vol.13 (3), 71-88.

Dweck, C. \& Leggett, E. (1988). A Social-cognitive Approach to Motivation and Personality. Psychological Review, vol.95 (2), 256-273.

Eccles, J. \& Wigfield, A. (2002). Motivational Beliefs, Values and Goals. In S. T. Fiske, D. L. Schacter \& C. Sahn-Waxler (Eds.), Annual Review of Psychology (pp. 109-132). Palo Alto, CA: Annual Reviews.

Elliot, A. J. \& Dweck, C. S. (2007). Handbook of Competence and Motivation. London: The Guilford Press.

., Shell, M. M., Henry, K. B. \& Maier, M. A. (2005). Achievement Goals, Performance Contingencies, and Performance Attainment: An Experimental Test. Journal of Educational Psychology, vol.97 (4), 630-640.

., Adler, T. F., Futterman, R., Goff, S. B., Kaczala, C. M., Meece, J. L. \& Midgley, C. (1983). Expectancies, Values, and Academic Behaviors. In J. T. Spence (Ed.), Achievement and Achievement Motivation (pp. 75-146). San Francisco, CA: W.H. Freeman and Company.

Feldman, R. S. \& Theiss, A. J. (1982). The Teacher and Student as Pygmalions: Joint Effects of Teacher and Student Expectations. Journal of Educational Psychology, vol.74 (2), 217-223.

. \& Prohaska, T. (1979). The Student as Pygmalion: Effect of Student Expectation on the Teacher. Journal of Educational Psychology, vol.71 (4), 485-493.

Flament, C. (2001). Structure, dynamique et transformations des représentations sociales. En J. C. Abric (Ed.), Pratiques sociales et représentations (pp. 37-58). Paris: Presses Universitaires de France.

García Bacete, F. y Doménech Betoret, F. (1997). Motivación, aprendizaje y rendimiento escolar. REME. Revista Electrónica de Motivación y Emoción, vol.1 (0). Recuperado desde http://reme.uji.es/ articulos/pa0001/texto.html

García, E. (1997). La naturaleza del conocimiento escolar: ¿Transición de lo cotidiano a lo científico o de lo simple a lo complejo? En M. J. Rodrigo y J. Arnay (Eds.), La construcción del conocimiento escolar (pp. 59-79). Barcelona: Paidós.

García López, J. (2006). Aportaciones de la teoría de las atribuciones causales a la comprensión de la motivación para el rendimiento escolar. Ensayos, (21), 217-232.

Gollwitzer, P. M., Oettingen, G., Smelser, N. J. \& Baltes, P. B. (2001). Motivation: History of the Concept. In N. J. Smelser \& P. B. Baltes (Eds.), International Encyclopedia of the Social and Behavioral Sciences (pp. 10109-10112). Oxford: Elsevier.

González Cabanach, R., Valle, A., Núñez, J. C. y González-Pienda, J. A. (1996). Una aproximación teórica al concepto de metas académicas y su relación con la motivación escolar. Psicothema, vol.8 (1), 45-61.

Huertas, J. (1997). Motivación: Querer aprender. Buenos Aires: Aique.

Long, J. F., Monoi, S., Harper, B., Knoblauch, D. \& Murphy, P. K. (2007). Academic Motivation 
and Achievement among Urban Adolescents. Urban Education, vol.42 (3), 196-222.

Matos, L. y Lens, W. (2006). La Teoría de Orientación a la Meta, estrategias de aprendizaje y rendimiento académico en estudiantes de secundaria de Lima. Persona, (9), 11-30.

MINEDUC (2003). Marco para la Buena Enseñanza. Santiago: Centro de Perfeccionamiento, Experimentación e Investigaciones Pedagógicas (CPEIP), Ministerio de Educación de Chile.

Miñano Pérez, P. y Castejón Costa, J. L. (2008). Capacidad predictiva de las variables cognitivomotivacionales sobre el rendimiento académico. REME. Revista Electrónica de Motivación y Emoción, vol.11 (28). Recuperado desde http://reme.uji.es/articulos/numero28/article4/article4.pdf

Miranda, A., Arlandis, P. y Soriano, M. (1997). Instrucción en estrategias y entrenamiento atribucional: Efectos sobre la resolución de problemas y el autoconcepto de los estudiantes con dificultades en el aprendizaje. Infancia y Aprendizaje, vol.20 (4), 37-52.

Murphy, P. \& Alexander, P. (2000). A Motivated Exploration of Motivation Terminology. Contemporary Educational Psychology, vol.25 (1), 3-53.

Neuville, S., Frenay, M. \& Bourgeois, É. (2007). Task Value, Self-efficacy and Goal Orientations: Impact on Self-regulated Learning, Choice and Performance among University Students. Psychologica Belgica, vol.47 (1), 95-117.

(2004). La perception de la valeur des activités d'apprentissage: Étude des déterminants et effets. Thèse de Doctorat. Louvain La Neuve: Université Catholique de Louvain.

, Bourgeois, É. \& Frenay, M. (2004). The Subjective Task Value: Clarification of a Construct. In S. Neuville (Ed.), La perception de la valeur des activités d'apprentissage: Étude des déterminants et effets. Thèse de Doctorat. Louvain La Neuve: Université Catholique de Louvain.

Nuttin, J. (1985). Théorie de la motivation humaine. Paris: Presses Universitaires de France.

Pajares, F. (1996). Self-efficacy Beliefs in Academic Settings. Review of Educational Research, vol.66 (4), 543-578.

Pelletier, L. et Vallerand, R. (1993). Une perspective humaniste de la motivation: Les théories de la compétence et de l'autodétermination. En R. Vallerand et E. Thill (Eds.), Introduction à la psychologie de la motivation (pp. 233-281). Laval: Editions Études Vivantes-Vigot.

Robbins, S., Lauver, K., Le, H., Davis, D., Langley, R. \& Carlstrom, A. (2004). Do Psychosocial and Study Skill Factors Predict College Outcomes? A Meta-analysis. Psychological Bulletin, vol.130 (2), 261-288.

Ryan, R. M. \& Deci, E. L. (2000). Self-determination Theory and the Facilitation of Intrinsic Motivation, Social Development, and Well-being. American Psychologist, vol.55 (1), 68-78.

Sansone, C. \& Harackiewiecz, J. (2000). Intrinsic and Extrinsic Motivation: The Search for Optimal Motivation and Performance. San Diego: Academic Press.

Schiefele, U. (1991). Interest, Learning, and Motivation. Educational Psychologist, vol.26 (3/4), 299-323.

Silva-Peña, I., Navarrete, P. y Zúñiga, M. J. (2012). Motivación escolar: La precepción de profesores en ejercicio y estudiantes de pedagogía. Informe Grupo Focal Fondecyt 1120351. Temuco: Universidad Católica de Temuco.

Silvia, P. J. (2006). Exploring the Psychology of Interest. Oxford: Oxford University Press.

Utman, C. H. (1997). Performance Effects of Motivational State: A Meta-analysis. Personality \& Social Psychology Review, vol.1 (2), 170-182.

Valenzuela, J. (2009). Características psicométricas de una escala para caracterizar el sentido del aprendizaje escolar. Universitas Psychologica, vol.8 (1), 49-59.

. (2007). Más allá de la tarea: Pistas para una redefinición del concepto de motivación escolar. Educaçao e Pesquisa, vol.33 (3), 409-426.

(2006). Enseñanza de habilidades de pensamiento y motivación escolar. Efectos del Modelo Integrado para el Aprendizaje Profundo (MIAP) sobre la motivación de logro, el sentido del aprendizaje escolar y la autoeficacia. Tesis doctoral. Santiago: Pontificia Universidad Católica de Chile. 
Vansteenkiste, M., Lens, W. \& Deci, E. L. (2006). Intrinsic versus Extrinsic Goal Contents in Self-determination Theory: Another Look at the Quality of Academic Motivation. Educational Psychologist, vol.41 (1), 19-31.

Weiner, B. (2008). Reflections on the History of Attribution, Theory and Research. Social Psychology, vol.39 (3), 151-156.

. (1990). History of Motivational Research in Education. Journal of Educational Psychology, vol.82 (4), 616-622. DOI: 10.1037/0022-0663.82.4.616

. (1985). An Attributional Theory of Achievement Motivation and Emotion. Psychological Review, vol.92 (4), 548-573.

Weinstein, R., Marshall, H., Sharp, L. \& Botkin, M. (1987). Pygmalion and the Student: Age and Classroom Differences in Children's Awareness of Teacher Expectations. Child Development, vol.58 (4), 1079-1093.

Wigfield, A. \& Cambria, J. (2010). Students' Achievement Values, Goal Orientations, and Interest: Definitions, Development, and Relations to Achievement Outcomes. Developmental Review, vol.30 (1), 1-35.

. \& Eccles, J. (2000). Expectancy-value Theory of Achievement Motivation. Contemporary

Educational Psychology, vol.25 (1), 68-81.

(1992). The Development of Achievement Task Values: A Theoretical Analysis.

Developmental Review, vol.12 (3), 265-310.

\& Roeser, R. (1998). Relations of Young Children's Ability related Beliefs to their Subjective Task Values, Performance, and Effort. Motivation and Affect in the Classroom. International Congress of Applied Psychology. San Francisco, United States.

(1994). Expectancy-value Theory of Achievement Motivation: A Developmental Perspective. Educational Psychology Review, vol.6 (1), $49-78$. 
